Aula Abierta

\title{
Estructura y contenidos de la comunicación y redacción de artículos científicos
}

\section{Structure and content of communication and writing scientific articles}

Jerónimo Maqueda Blasco, M. ${ }^{a}$ Fe Gamo González, Rosana Cortés Barragán, Jorge Veiga-Cabo

Escuela Nacional de Medicina del Trabajo, Instituto de Salud Carlos III, Madrid. España.

\section{Recibido: 15-01-13}

Aceptado: 20-02-13

\section{Correspondencia}

Jerónimo Maqueda Blasco

Escuela Nacional de Medicina del Trabajo

Instituto de Salud Carlos III

Pabellón 8, Ciudad Universitaria

28040 Madrid. España.

Tfno: 918224013

jmaqueda@isciii.es

\section{Resumen}

La comunicación científica es parte del proceso investigador y representa unos de los retornos que recibe la Sociedad del investigador. La comunicación científica se somete a una estructura internacionalmente aceptada: Introducción, Material y Métodos, Resultados, Conclusiones y Discusión, en la que cada apartado tiene una finalidad y una intención. Tras haber realizado una revisión de las publicaciones y guías más recientes sobre comunicación científica en salud se ha sintetizado las recomendaciones más relevantes con la intención de orientar a los autores noveles en la redacción de sus primeras publicaciones.

Med Segur Trab (Internet) 2013; 59 (230) 159-170

Palabras clave: Comunicación científica, publicación científica.

\section{Abstract}

Scientific communication is part of the research process and represents one of the returns that Society receives from researchers. Scientific communication is under an internationally accepted structure: Introduction, Material and Methods, Results, Conclusions and Discussion, each item have a concrete purpose and intention. After has conducted a review of the most recent publications and guidelines on health science communication we have synthesized the most relevant recommendations in order to guide early scientific authors in write his scientific papers.

Med Segur Trab (Internet) 2013; 59 (230) 159-170

Key words: Scientific communication, scientific publication. 


\section{INTRODUCCIÓN}

El término "comunicación" procede etimológicamente del latín "comunicare” que significa compartir o poner en común, y la Comunicación Científica, como una parte específica de ella, es parte del proceso investigador (figura 1) en cuanto que constituye el producto final, derivado de sus resultados y representa la vía de retorno de conocimiento a la comunidad científica y a la Sociedad tras el esfuerzo investigador. Podríamos considerar este producto final de la investigación en forma de comunicación científica, como un deber ético del investigador en ese compromiso de compartir los avances en el conocimiento con el resto de la comunidad científica.

Figura 1. Etapas del Proceso de Investigación

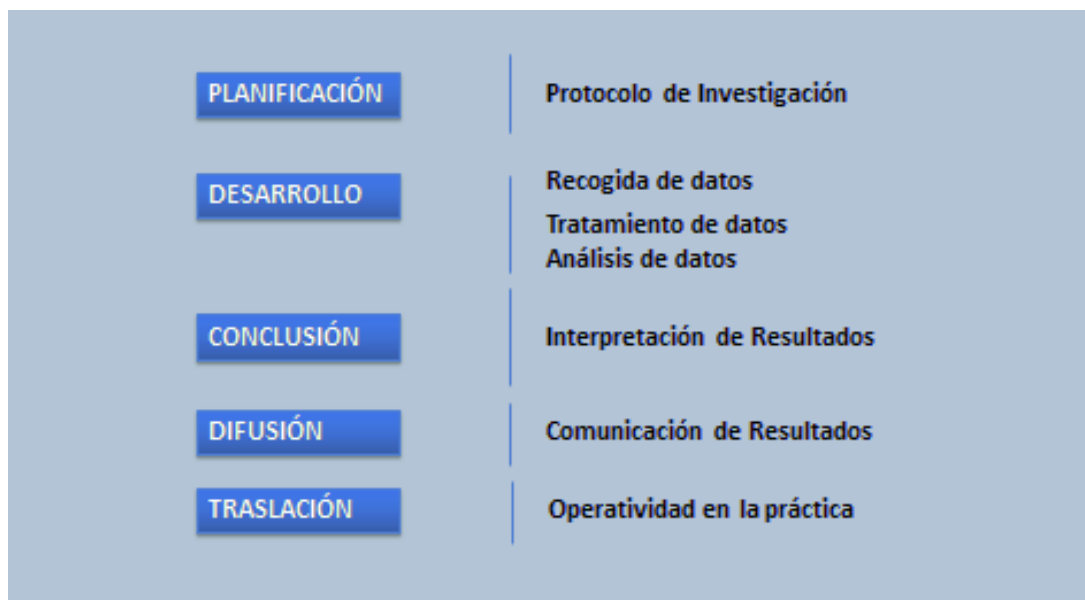

Mabmoud F. Fathalla en "A Practical Guide for Health Researchers" editada por $O M S^{1}$, considera que la investigación no se completa hasta que se escribe y se comparten sus resultados no sólo con otros científicos, sino también con aquellos que puedan utilizarlos o beneficiarse de ellos.

La Comunicación Científica varía en forma y contenido en función del sector hacia el que se dirige, así si los resultados se dirigen a la Sociedad en general, se habla de Divulgación Científica. Si se dirigen a los profesionales, la comunicación puede adoptar la forma de Guías de Buenas Prácticas, Protocolos o Procedimientos. Si nos dirigimos a la Comunidad Científica lo haremos en forma de Artículo Científico, Comunicación oral o Poster Científico. A las Agencias Financiadoras se debe presentar en forma de Proyecto de Investigación y cuando el destinatario es el Estamento de Toma de Decisiones la Comunicación adopta una estructura de Informe.

\section{Principios generales de la comunicación científica}

Según la UNESCO "la finalidad esencial de un artículo científico es comunicar los resultados de investigaciones, ideas y debates de una manera clara, concisa y fidedigna" 2

La calidad del proyecto y de su ejecución son los determinantes de un buen artículo científico, sin calidad en el diseño de la investigación, sistematización en el trabajo de campo, estandarización de la información, rigor analítico y reflexión sobre los resultados no es posible desarrollar una publicación de calidad.

Debemos tener en cuenta que escribir un artículo científico es un proceso que requiere tiempo, dedicación y esfuerzo. En cuanto al estilo de redacción, existen unas normas y características propias de la comunicación científica y es muy importante seguir unos principios generales:

- Transparencia: en el sentido en el que podríamos considerar la comunicación científica como un acto "notarial" que debe reflejar fielmente el trabajo realizado (desde términos de búsqueda a métodos analíticos, estadísticos, etc.). No deben 
obviarse los resultados negativos (corroboración o no de nuestras hipótesis o consecución o no de nuestros objetivos).

- Reproducibilidad: debe tener un nivel suficiente de exhaustividad y detalle tal que permita a otros grupos reproducir nuestra investigación.

- Coherencia Argumental: respondiendo a un argumento construido entorno a la Hipótesis y los Objetivos, de forma que los diferentes apartados que componen la estructura de un artículo científico giren en torno a ellos. La Introducción debe responder al estado actual del arte en función de las hipótesis y objetivos planteados y por lo tanto justificar su formulación. La Metodología debe ir dirigida al contraste de la hipótesis y la consecución de los objetivos, los Resultados deben proceder de los análisis realizados y la Conclusiones deben precisar y se acepta o rechaza nuestra hipótesis y si se han conseguido o no nuestros objetivos (figura 2).

Figura 2. Etapas del Proceso de Investigación

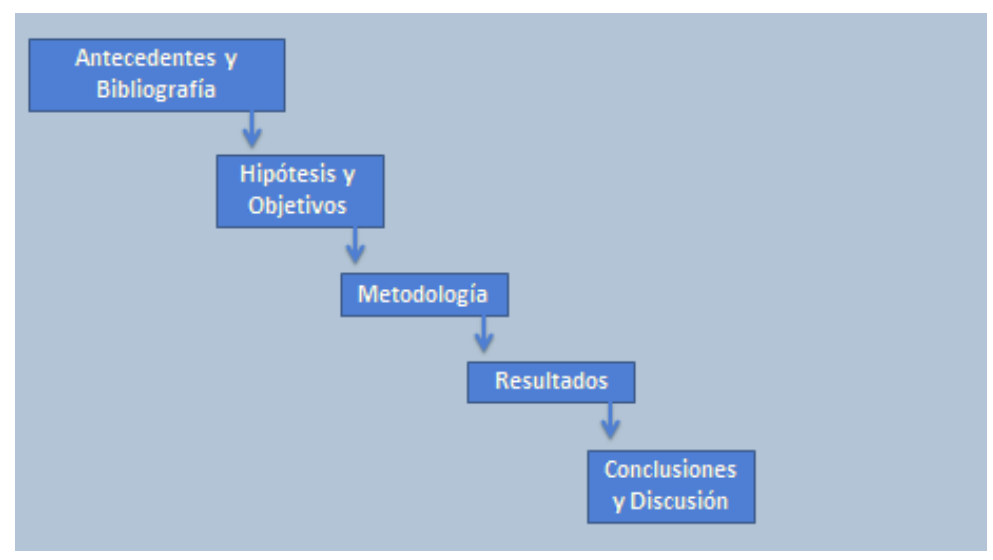

- Autocrítica: Deben comunicarse las dificultades encontradas, las limitaciones que tienen nuestros resultados y la dirección de los mismos en favor o en contra de nuestras hipótesis y objetivos.

- Valorización de resultados: Debe expresarse la aportación que suponen nuestros resultados y conclusiones a la mejora del conocimiento del Estado del Arte. Un trabajo es publicable en la medida que por sus conclusiones, resultados, métodos o análisis realizados añadan conocimiento o aporten soluciones a una pregunta sin resolver en la práctica diaria.

- Lenguaje: Pierson $D J^{3}$ y Webe $E J^{4}$ estiman que el $44 \%$ de los artículos que se remiten a revistas científicas nunca serán publicados. Las causas de rechazo, en muchas ocasiones se relacionan con el estilo de redacción ${ }^{5,6}$, lenguaje abigarrado, abuso de abreviaturas, etc. El estilo más universal es el estilo sencillo y directo (Claro, Preciso y Conciso). Campos $\mathrm{Rosa}^{7}$ define las características del lenguaje científico, enfrentándole al estilo del lenguaje literario (tabla I).

Tabla I. Diferencias de estilo en la redacción de un artículo científico

\begin{tabular}{ll}
\hline \multicolumn{1}{c}{ ESTILO CIENTÍFICO } & \multicolumn{1}{c}{ ESTILO LITERARIO } \\
\hline Informativo & Entretenido \\
\hline Racional & Emotivo \\
\hline Impersonal & Personal \\
\hline Objetivo & Subjetivo \\
\hline Concisión y claridad de datos & Originalidad de estilo \\
\hline Presenta una realidad vivida & Crea una realidad no vivida \\
\hline
\end{tabular}

Fuente: Campos Rosa J. 
En términos anglosajones el estilo debe responder al acrónimo "KISS" (Keep It Simple and Short).

En relación al manejo de la redacción debe ser impersonal o tercera persona y el tiempo verbal varía en función del apartado, en la tabla II se sintetizan las recomendaciones de Hernández Meléndrez ${ }^{8}$.

Tabla II. Manejo del tiempo verbal de acuerdo al apartado o sección del artículo

\begin{tabular}{lll}
\hline \multicolumn{1}{c}{ APARTADO O SECCIÓN } & \multicolumn{1}{c}{ CONTENIDOS } & $\begin{array}{r}\text { TIEMPO } \\
\text { VERBAL }\end{array}$ \\
\hline Resumen & $\begin{array}{l}\text { Refleja una síntesis de lo } \\
\text { realizado }\end{array}$ & Pasado \\
\hline Introducción & $\begin{array}{l}\text { Refleja el estado actual } \\
\text { del conocimiento }\end{array}$ & Presente \\
\hline Material y Métodos & $\begin{array}{l}\text { Describe lo que se } \\
\text { estudió, como se estudió } \\
\text { y como se analizó }\end{array}$ & Pasado \\
\hline Resultados & $\begin{array}{l}\text { Refleja los hallazgos } \\
\text { encontrados }\end{array}$ & Pasado \\
\hline Conclusión y Discusión & $\begin{array}{l}\text { Describe la aportación } \\
\text { de nuestro estudio al } \\
\text { conocimiento existente }\end{array}$ & Presente \\
\hline
\end{tabular}

Fuente: Hernández Meléndrez.

Además de lo expuesto y como en todo, la experiencia es uno de los factores más importantes a la hora de adquirir soltura en la forma de redacción, pero independientemente de nuestra experiencia y habilidades innatas para escribir, la lectura habitual y sistemática de artículos científicos publicados en revistas de calidad, es uno de los elementos más importantes a la hora de adquirir un estilo adecuado de redacción científica o mejorarlo.

Este trabajo pretende orientar a los profesionales de Medicina del Trabajo y en general, del ámbito de las ciencias biomédicas, en la redacción y elaboración de manuscritos científicos, facilitando una serie de pautas y recomendaciones que a modo de guía, sirvan para mejorar la calidad de la comunicación y, en lo posible, los índices de aceptación de sus artículos originales o cualquier otra modalidad de publicación científica.

\section{Estructura de un Artículo Científico}

El International Committee of Medical Journal Editors ${ }^{9}$, reconoce una estructura genérica del Manuscrito Científico que recoge los siguientes apartados: Introducción, Métodos, Resultados y Discusión “IMRAD”, que responden a las preguntas que Bradford Hill considera que debe responder una publicación científica (tabla III).

Tabla III. Estructura genérica de un artículo científico

\begin{tabular}{ll}
\hline \multicolumn{1}{c}{$\begin{array}{c}\text { APARTADOS DE UN ARTÍCULO } \\
\text { CIENTífICO }\end{array}$} & \multicolumn{1}{c}{$\begin{array}{c}\text { PREGUNTAS DE BRADFORD HILL } \\
\text { (1965) }\end{array}$} \\
\hline Introducción & ¿Porqué se hizo? \\
\hline Métodos & ¿Qué se hizo? \\
\hline Resultados & ¿Qué se encontró? \\
\hline Discusión & ¿Qué significan los resultados? \\
\hline
\end{tabular}

International Committee of Medical Journal Editors.

1. Título en una Comunicación Científica: Podemos considerarlo como la "tarjeta de presentación del trabajo" frente al lector investigador o miembro de la 
comunicad científica. El título es lo primero que se lee en una publicación y por tanto condiciona la invitación o rechazo a que sea leído. ${ }^{10}$

Diferentes autores y guías de redacción científica coinciden en las características que hacen a un título ser adecuado:

- Debe describir los contenidos de forma concreta y aunque no existe una norma concreta sobre su tamaño, ha de prevalecer la idea de que no sea excesivamente largo y se suele establecer un límite de entre 10 a 15 palabras.

- Debe ser específico o conciso en relación al tema tratado, evitando términos genéricos.

- Evitar la utilización de términos como: Estudio, Investigación, Análisis, etc., que son superfluas porque se supone que son parte del trabajo como artículo original.

- No deben aparecer acrónimos ni abreviaturas.

Se trata por lo tanto de describir lo más fielmente el contenido del artículo con el menor número posible de palabras, por eso es importante que una vez finalizado el artículo, repasemos el título para comprobar que refleja de forma escueta el contenido real del artículo o si por el contrario, necesita alguna adaptación una vez que tengamos la visión global del trabajo finalizado.

2. Autores: El International Committee of Medical Journal Editors establece que cada uno de los autores firmantes de un artículo deben haber contribuido suficientemente en el trabajo desde su diseño, recogida y análisis de datos, redacción o revisión del manuscrito y ser capaz de asumir la responsabilidad del mismo. Por lo general, el primer firmante debe corresponderse con el autor principal y el que ha llevado tanto el peso principal de la investigación como de la elaboración del artículo. El resto de autores pueden figuran en orden correlativo en cuanto a su contribución o por acuerdo dentro del equipo, soliendo firmar en último lugar el coordinador, supervisor o jefe de departamento.

Muchas revistas exigen que uno de los autores sea el encargado de recibir la correspondencia con otros científicos o lectores que se pueda derivar de la publicación e interés por el artículo. Este puede ser el autor principal, el coordinador o cualquiera de ellos que se acuerde por consenso.

Es importante adoptar desde el principio un formato que nos identifique siempre y de la misma forma como autor en cualquier artículo que firmemos y permita rescatar esta información desde cualquier base de datos bibliográfica.

En aquellos países en los que el matrimonio supone que la mujer abandone su apellido adoptando el de su marido cuando se casa, con frecuencia surge un problema en aquellas circunstancias en las que tras una ruptura de la relación y se vuelve a recuperar la condición inicial del apellido de soltera o se suceden otros matrimonios, por lo que es preferible definir desde el principio el nombre de autora que deberá definir siempre su carrera profesional.

En el caso de países como España, en los que se utilizan dos apellidos, uno procedente del padre y otro de la madre, el problema se suele presentar a la hora de indizar la autoría en bases de datos bibliográficas internacionales, en las que en su mayoría sólo contemplan la posibilidad de clasificar y recuperar por un solo apellido. En este caso, podemos optar por considerar un solo apellido (ej.: Juan Ledesma o Ledesma J, según las normas de cada base de datos) o en el caso de que prefiramos figuren los dos apellidos, se recomienda presentarlos separados por un guion (ej.: Juan Ledesma-Fernández o Ledesma-Fernández J).

3. Redacción del Resumen y Abstract: El Resumen, y su versión en inglés o Abstract, es relevante en términos de visibilidad del artículo y calidad de almacenamiento de información en documentación científica, pues permite al lector ampliar la información sobre los contenidos que aporta el título. Cuando un investigador realiza una búsqueda en diferentes bases de datos bibliográficas, 
lo primero que obtiene es una colección de títulos y resúmenes de los artículos relacionados con el tema objeto de estudio, y en base a esa información inicial debe establecer su decisión de incluir o no en su estudio cada uno de los artículos, y por tanto obtenerlos a texto completo.

De esto se podemos deducir la importancia que representa facilitar una información sintética pero completa de la investigación realizada a través del resumen, incluyendo el propósito de la investigación, los procedimientos o métodos utilizados, los principales resultados con información cuantitativa, estadística y su grado de significación, así como y las conclusiones más relevantes.

Por sus contenidos, un buen Resumen debe tener una autonomía con respecto al texto central y debe tener sentido por si mismo, sin necesidad de consultar el texto completo del artículo. No debe tener referencia a imágenes, tablas o bibliografía y tampoco deben figurar acrónimos o abreviaturas salvo que estas sean universalmente reconocidas. Determinadas revistas ${ }^{11}$ solicitan que el Resumen se encuentre estructurado en sub-apartados IMRAD (Introducción, Material y Métodos, Resultados, Conclusiones y Discusión), y aunque esta estructura no es siempre obligatoria, ayuda a organizar los contenidos.

No debe ser ni muy escueto ni excesivamente largo, en ocasiones algunas revistas acotan el número máximo de palabras del Resumen. En cualquier caso, podríamos tomar como referencia una extensión óptima que oscilase entre 150 a 250 palabras.

Es importante también tener en cuenta que el Abstract, como la versión en inglés del Resumen, ha de ser un fiel reflejo del mismo contenido de este y que debemos cuidar al máximo la calidad de la traducción.

Se recomienda que tanto el Título como el Resumen, se elaboren una vez finalizado el artículo, pues esto permite tener una visión más amplia del trabajo y ayuda a seleccionar los aspectos clave que deben figurar tanto en el Título como en el resumen.

4. Las Palabras Clave (Key Words): determinan en gran medida las condiciones de recuperación en las estrategias de búsqueda bibliográfica, de ahí su importancia. El mayor rendimiento en la recuperación de artículos se obtiene cuando utilizamos los mismos descriptores que utilizan los tesauros para la clasificación de los artículos a la hora de incluirlos en las bases de datos bibliográficas, por lo que si definimos nuestras palabras clave o Key Words utilizando los mismos términos utilizados por los tesauros, mejoraremos las posibilidades de recuperación posteriores de nuestros artículos por otros autores.

Entre los tesauros más utilizados en ciencias biomédicas podemos citar "Medical Subject Heading (MeSH)" que es el que utiliza la National Library of Medicine (NLM) de EEUU como motor de búsqueda en sus bases de datos bibliográficas, MEDLINE y PubMed.

Su versión traducida al español y al portugués es "Descriptores en Ciencias de la Salud (DeCS)", que constituye el motor de búsqueda de las Bases de Datos LILACS (Literatura Latino Americana en Ciencias de la Salud) e IBECS (Índice Bibliográfico Español en Ciencias de la Salud). Ambos tesauros, MeSH y DeCS, permiten localizar el mismo término con su traducción exacta y de sus sinónimos en cualquiera de los tres idiomas (inglés, español y portugués), así como compartir cualquier estrategia de búsqueda desarrollada independientemente que la hayamos desarrollado sobre términos MeSH o DeCS.

The Cochrane Library utiliza también MeSH en su versión original en inglés aunque por el momento, aún se encuentran trabajando para poder utilizar $\mathrm{MeSH}$ $D e C S$ en su versión traducida al español.

EMBASE utiliza el tesauro EMTREE y otras bases de datos como OSH Update (contenidos específicos en temas de toxicología y de salud laboral), Web of Knowledge (WOK) y Scopus, no utilizan tesauros, aunque el definir nuestra estrategia de búsqueda utilizando términos $M e S H$-DeCS siempre nos ayudará en 
la recuperación de artículos en estas otras bases de datos, por la probabilidad de coincidencias en la terminología de clasificación y recuperación que pueda haber con las búsquedas sobre terminología libre.

5. Filiaciones: Es también muy importante que la institución o instituciones a las que pertenecen los autores se encuentren bien referidas, y a ser posible, citarlas siempre de la misma forma para que sean fácilmente identificables como la misma institución, independientemente del artículo o revista en las que hayan sido mencionadas

6. Introducción: La introducción establece el contexto del estado de conocimiento que se dispone en la actualidad sobre el tema de investigación, apuntando las lagunas de conocimiento existentes y hacia cuales de éstas se dirige nuestra investigación y trata de despertar el interés por la publicación.

No debe ser excesivamente larga que puedan aburrir y acaparar demasiada atención al lector respecto a otros apartados de más peso y de la estructura central del artículo. Este apartado se construye en base a una secuencia argumental que se refleja en la figura 3 y en la que es importante tener en cuenta los siguientes aspectos:

- No debe haber una "sobre-referencia", las citas bibliográficas deben ser las necesarias y deben ser actuales (entre 6 y 10 años dependiendo del tema, considerándose lo ideal por debajo de los 6 años), salvo publicaciones que constituyan "hitos", en la evolución del conocimiento del tema investigado.

- No deben figurar ni datos ni afirmaciones que posteriormente aparezcan en los apartados de "Resultados" o "Conclusiones".

- No debe extenderse en conocimientos ya consolidados.

Figura 3. Secuencia argumental en el apartado de Introducción

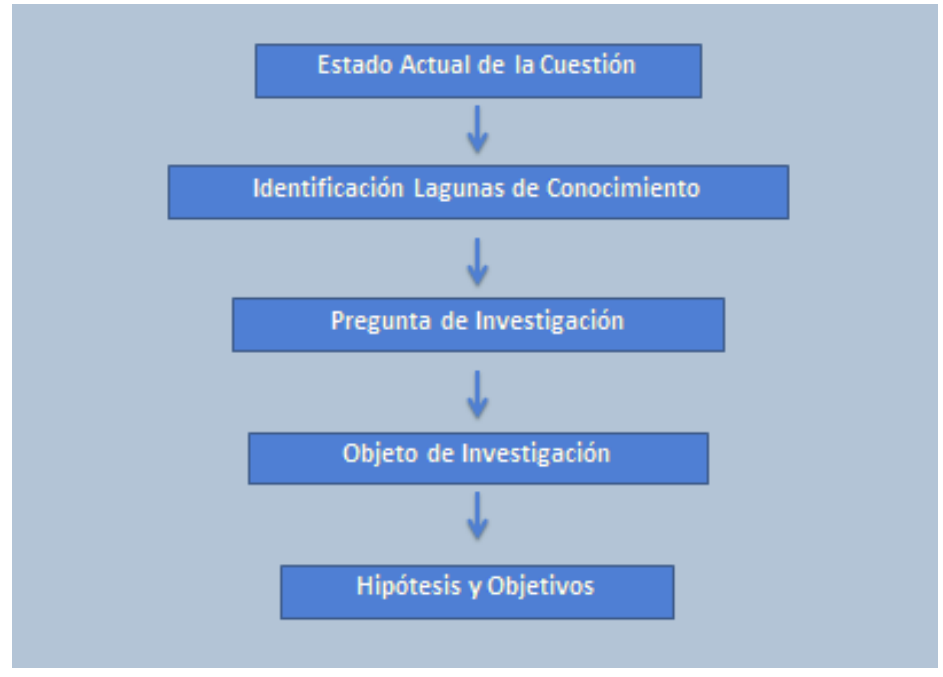

7. Hipótesis y Objetivos: Bien puede ser objeto de desarrollo en un epígrafe propio, como ocurre en los proyectos de investigación o incorporarlo dentro de la redacción del artículo como párrafo final de Introducción.

En relación a la/s hipótesis deben considerarse los siguientes aspectos:

- La hipótesis representa la teoría del investigador en relación al tema de investigación (no es una pregunta de investigación).

- La hipótesis debe formularse de forma directa y sencilla en un texto breve (no se pueden formular varias hipótesis en un mismo párrafo).

- Las hipótesis se contrastan, por lo que condicionan el diseño, metodología y análisis (lo que no es contrastable no es una hipótesis). 
- Los tipos de hipótesis condicionan el diseño del estudio, siendo las más frecuentes: Hipótesis de Asociación, Hipótesis de Causalidad, Hipótesis de diferencia.

Con respecto a la redacción de los objetivos hay que considerar:

- Representan los alcances o logros del proyecto de investigación.

- Los objetivos se alcanzan o se consiguen (los logros no alcanzables no son objetivos).

- Determinan tanto el diseño como los aspectos metodológicos y analíticos.

- Deben expresarse en términos de conocimiento: Conocer, Estimar, Identificar, Validar.

- Deben eludirse términos como: Estudiar, Analizar, Investigar .

- Pueden suponer bien un "hito final" (Objetivo Principal, General) alcanzable mediante del desarrollo completo de la investigación, o "hitos intermedios" (Objetivos Secundarios, Específicos), alcanzables durante el desarrollo del proyecto o sub-productos del mismo

8. Material y Métodos: Desde el punto de vista científico este apartado es el más complejo y representa el "Core" de la publicación científica, determinando tanto la validez interna como externa de los resultados.

Hay dos aspectos críticos a la hora de redactar este apartado:

a. La redacción de este apartado debe ser tal que permita a otros investigadores repetir el estudio de cara a reproducir los resultados.

b. Debe ser acorde con las hipótesis y objetivos formulados.

Este apartado debe cumplir las siguientes condiciones:

- Deben describirse de forma detallada los aspectos relacionados con el diseño: tipo de estudio, muestra, error muestral, control de sesgos, métodos de medida, fuente de datos, periodo de recogida de datos, reclutamiento, seguimiento, etc.

- En el caso de utilizar métodos ya estandarizados o validados sólo es necesaria su mención no debiéndose describir de forma detallada.

- Especificarse los aspectos éticos y de confidencialidad.

- Los métodos estadísticos deben describirse con un nivel de detalle que permitan reproducir los análisis en el caso de acceder a los micro datos.

- Evitar la utilización, fuera del ámbito estadístico, términos como "significativo", "correlación", "relación", "asociación", etc.

- En los resultados estadísticos especificar el valor "p" de corte.

9. Resultados: En la redacción de este apartado el investigador tiene que tener siempre presente las hipótesis y objetivos formulados. Este apartado es la base para expresar las evidencias obtenidas.

Los principales aspectos a considerar son:

- Tiene que estar dirigido hacia la verificación de la hipótesis y a la consecución de los objetivos.

- No deben expresarse resultados no procedentes del análisis de los datos.

- Deben expresarse tanto los resultados positivos como negativos.

- Debe resumirse la información mediante la utilización de tablas.

- Las figuras deben utilizarse para enfatizar resultados (no son buenas herramientas para expresar información numérica exacta).

- En general, debe utilizarse numeración romana para las tablas (Tabla I, Tabla II, etc.) y arábiga para las figuras (Figura 1, Figura 2, etc.).

- Evitar, en el texto, la repetición de datos expresados en tablas o figuras. 
- Las tablas y figuras deben estar referenciadas en el texto.

- Las tablas deben elaborarse de tal forma que para su lectura no sea necesario ir al texto (título, cabecera de filas, cabecera de columnas, unidades de medida, significados de abreviaturas, fuente de los datos, etc.).

- No deben expresarse conclusiones.

10. Discusión y Conclusiones: Este apartado representa la interpretación intelectual de los resultados obtenidos, por lo tanto el autor no debe expresar como conclusiones aquellas que no se deriven directamente de los resultados. Debemos discutir nuestros resultados de forma que el autor debe expresar:

- La respuesta a la pregunta de investigación.

- Concretar las evidencias encontradas.

- Expresar la verificación o no de las hipótesis y la consecución o no de los objetivos formulados.

- Enfatizar los principales hallazgos.

- Enfrentar nuestros resultados con otras publicaciones científicas.

- Exponer las debilidades del estudio y sus limitaciones en cuanto al alcance de los resultados (evaluación crítica de los autores).

- Analizar las causas potenciales de los resultados negativos.

- Formular preguntas para investigaciones posteriores.

A partir de esta discusión sacaremos nuestras conclusiones, que han de ser interpretativas, no descriptivas, por lo tanto no pueden reproducir datos cuantitativos y es importante que realicemos un esfuerzo por resumir las principales aplicaciones o beneficios en términos de salud que nuestro trabajo pueda reportar.

11. Referencias Bibliográficas o Bibliografía: Se trata de un apartado en el que se encuentran relacionadas las citas bibliográficas utilizadas para el trabajo, por el mismo orden en el que han ido apareciendo su referencia en el texto como se indicó anteriormente. Este apartado refleja la base documental en la que se ha asentado la investigación y apoya principalmente los apartados de introducción, metodología y discusión.

Al igual que en el apartado de Material y Método se insistía sobre la importancia de que su redacción debía ser tal que permita a otros investigadores repetir el estudio de cara a reproducir los resultados, el apartado de Bibliografía, aunque no requiere ningún esfuerzo de redacción por tratarse de una mera relación de trabajos, exige un cuidado especial por seguir unos requisitos universales de citación que permitan a cualquier lector recuperar alguno de los artículos o trabajos referenciados en nuestro artículo en caso de que los necesite.

Para referenciar correctamente cada uno de los trabajos que hemos utilizado y citados en el texto, debemos utilizar los Requisitos de Uniformidad para Manuscritos, lo que conocemos como Normas de Vancouver por tratarse de una evolución de las mismas, y que son establecidas por el Comité Internacional de Directores de Revistas Médicas (International Commitee of Medical Journal Editors, http://www.icmje.org/).

La bibliografía debe ser reciente, como orientación y como se mencionó anteriormente, podemos centrarnos en bibliografía que se encuentre publicada en los últimos seis años, pero como también se ha mencionado, este aspecto depende del tema concreto que estemos estudiando y en ocasiones nos vemos obligados a ampliar el periodo de estudio, en ocasiones para alcanzar un número razonable de artículos para la revisión y en otras por diferentes motivos. También debemos procurar que sean adecuadas y se ajusten con criterios de pertinencia al tema de investigación propuesto. Respecto al número de referencias debe ser razonable y raramente debiera superar las 40 citas (Hasley 1998). 
La gestión de la bibliografía es una tarea complicada, por ello es importante la utilización de gestores de información desde el inicio de la búsqueda bibliográfica algunos de ellos de libre utilización.

12. Anexos: En los anexos de deberá incluir aquel material suplementario que se considere necesario adjuntar para mejorar la comprensión del trabajo (encuestas, relación de enfermedades u otras, test utilizados, resultados analíticos, tablas de valores, etc.).

13. Menciones especiales, agradecimientos y colaboraciones: En esta sección se hará mención especial cuando el trabajo sea producto de un proyecto económicamente soportado por alguna Agencia de Financiación, organismo o institución, pública o privada, detallando en lo posible el número de proyecto que le fuera asignado. En ocasiones, este es un requisito exigido por las Agencias Financiadoras respecto a todos los productos derivados de la financiación de un proyecto y es importante tenerlo siempre en cuenta.

En ocasiones en las que algunas personas o instituciones han contribuido de forma especial al desarrollo del trabajo, sin legar a alcanzar el nivel de autoría, se puede utilizar el agradecimiento o mención especial a la contribución en la realización del estudio. Es importante tener en cuenta que siempre que se haga mención a personas físicas o jurídicas incluidas en este apartado, deberán tenerse el conocimiento y consentimiento para dicha mención, correspondiendo a los autores la gestión y responsabilidad de cumplimiento de dicho trámite y requisito.

14. Autorizaciones: Cuando se reproduzca alguna imagen obtenidas de otras publicaciones, Internet, etc., debemos comprobar que estas no se encuentran protegidas por copyright y que se encuentran bajo licencia "Open Access" "Creative Commons"13, permitiendo así su reproducción. En caso de que se necesite autorización previa para su publicación, será responsabilidad de los autores la obtención de la misma.

\section{Calidad de los estudios}

El valor y validez de la Comunicación Científica está condicionado por la calidad de cada una de las fases del proceso de investigación y se inicia en la elaboración del proyecto de investigación en un proceso secuenciado, de tal forma que la calidad de una determinada etapa, depende de la calidad de la etapa anterior.

La evaluación de la calidad de un artículo es una preocupación de los editores de las revista científicas. En este contexto surge en 2004 la Iniciativa STROBE (Strengthening the Reporting of Observational studies in Epidemiology $)^{14}$, esta iniciativa tiene un especial interés en Medicina del Trabajo dado que los diseños observacionales son los más frecuentemente utilizados en nuestro ámbito.

La iniciativa STROBE reúne a editores de las principales revistas médicas (Annals of Internal Medicine, BMJ, Bulletin of the World Health Organization, International Journal of Epidemiology, JAMA, Preventive Medicine y The Lancet), epidemiólogos, metodólogos, estadísticos y clínicos.

La iniciativa STROBE acuerda 22 puntos de evaluación para valorar sobre la calidad de una comunicación científica como herramienta puesta a disposición de autores, editores, revisores y lectores de artículos científicos (tabla IV). 
Tabla IV. Número de ítems de evaluación y apartado de un artículo científico. Declaración STROBRE

\begin{tabular}{|c|c|c|}
\hline APARTADO & $\begin{array}{l}\mathbf{N}^{\circ} \text { del } \\
\text { Ítem }\end{array}$ & CONCEPTO EVALUADO \\
\hline Título y el resumen & 1 & \\
\hline \multirow{2}{*}{ Introducción } & 2 & Contexto/fundamentos \\
\hline & 3 & Objetivos \\
\hline \multirow{9}{*}{ Metodología } & 4 & Diseño del estudio \\
\hline & 5 & Contexto \\
\hline & 6 & Participantes \\
\hline & 7 & Variables \\
\hline & 8 & Fuentes de datos/medidas \\
\hline & 9 & Sesgos \\
\hline & 10 & Tamaño muestral \\
\hline & 11 & Variables cuantitativas \\
\hline & 12 & Métodos estadísticos \\
\hline \multirow{4}{*}{ Resultados } & 13 & Participantes \\
\hline & 14 & Datos descriptivos \\
\hline & 15 & Datos de las variables de resultado \\
\hline & 16 & Resultados principales \\
\hline Otros análisis & 17 & \\
\hline \multirow{4}{*}{ Discusión } & 18 & Resultados clave \\
\hline & 19 & Limitaciones \\
\hline & 20 & Interpretación \\
\hline & 21 & Generabilidad \\
\hline Financiación & 22 & \\
\hline
\end{tabular}

\section{REFERENCIAS BIBLIOGRÁFICAS}

1. Mahmoud F. Fathalla. A Practical Guide for Health Reaserchers [Internet]. Cairo: WHO. 2004 [citado 2012 nov 1]. Disponible en http://applications.emro.who.int/dsaf/dsa237.pdf.

2. UNESCO. Programa General de Información y UNISIT. Guía de Redacción de artículos científicos destinados a la publicación. París. 1983 May. 13 p.

3. Pierson DJ. The Top Ten Reasons Why Manuscripts Are Not Accepted Publication. Respir Care. 2004; 49(10): 1246-52

4. Weber EJ, Callaham ML, Wears RL, Barton C, Young G. Unpublished Research from a Medical Specialty Meeting. J of Surg Research 2005; 128: 165-7.

5. Bordage G. Reasons Reviewrs Reject and Accept Manuscripts: The Strengthsand Weakness in Medical Education Reports. Acad Med. 2001 Sep; 76(9):889-96.

6. Vancouver Group. Errores en los Manuscritos Enviados a Publicación: A propósito de los requerimientos. Rev Mex Pediatr. 1998; 65(1): 2-4.

7. Campos Rosa J. La Comunicación científica: ¿arte o técnica? Ars Pharmaceutica.2000; 41(1): 11-18.

8. Edelsys Hernández Meléndrez. Cómo escribir una tesis [Internet]. Valparaíso. Universidad Pontificia Católica. 2006 [citado 2012 nov 1]. Disponible en: http://biblioteca.ucv.cl/site/servicios/documentos/ como_escribir_tesis.pdf. 
9. International Committee of Medical Journal Editor. Uniform Requirements for Manuscripts Submitted to Biomedical Journals: Writing and Editing for Biomedical Publication [Internet]. ICMJE 2010 [citado 2012 nov 1]. Disponible en http://www.icmje.org/urm_full.pdf.

10. Reyna Lucía Elizondo-Rivera, Francisco Javier Bosques-Padilla. Cómo escribir un artículo científico: cómo lograr que su investigación sea publicada. Rev Gastroenterol Mex. 2007; 72 (2): 113-6.

11. Journals.lww.com [Internet]: Journal of Occupational and Environmental Medicine; [cited 2012 Nov 1]. Journal of Occupational and Environmental Medicine Online Submission and Review System. Disponible en http://edmgr.ovid.com/joem/accounts/ifauth.htm.

12. Saenz-Valero J, D’Agostino MJ, Castiel LD, Veiga de Cabo J. La Iniciativa Open Access. Una Visión de Conjunto. Med Segur Trab. 2007; 53(207):5-10.

13. Veiga de Cabo J. La revista Medicina y Seguridad en el Trabajo y el respeto al derecho de autoría. Med Segur Trab (Internet). 2012; 58:(228) 174-178.

14. Erik von Elm, Douglas G. Altman, Matthias Egger, Stuart J. Pocock, Peter C. Gotzsche, Jan P. Vandenbroucke; Iniciativa STROBE. Declaración de la Iniciativa STROBE (Strengthening the Reporting of Observational studies in Epidemiology): directrices para la comunicación de estudios observacionales. Gac Sanit. 2008; 22(2):144-50.

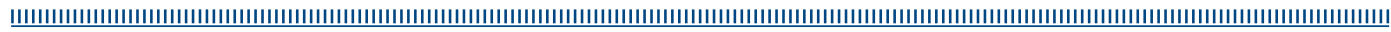

